\title{
External or subcomissural annuloplasty in bicuspid aortic valve repair: which is better?
}

\author{
Metesh Acharya ${ }^{1}$, Hiwa Sherzad ${ }^{1}$, Sven Zhen Cian Tan $^{2}$, Mohamad Bashir ${ }^{3}$, and Giovanni \\ Mariscalco $^{1}$ \\ ${ }^{1}$ Glenfield Hospital \\ ${ }^{2}$ Queen Mary University of London Barts and The London School of Medicine and \\ Dentistry \\ ${ }^{3}$ NHS Wales Health Education and Improvement Wales
}

November 12, 2021

\begin{abstract}
Patients with a bicuspid aortic valve (BAV) are at increased risk of valvular regurgitation compared to their counterparts with a tri-leaflet aortic valve. There is now increasing emphasis to offer BAV repair to mitigate the risks of prosthesis-related complications, including thromboembolism, haemorrhage and endocarditis, as well as structural valve deterioration and future re-operation with conventional valve replacement, particularly in younger populations. Furthermore, over the preceding two decades, our greater understanding of the functional anatomy of the BAV, pathophysiological mechanisms of BAV insufficiency and the development of a functional classification of aortic regurgitation have significantly contributed to the evolution of aortic valve reconstructive surgery. In this commentary, we discuss a recent article from the Journal of Cardiac Surgery comparing external annuloplasty and subcommissural annuloplasty as techniques for BAV repair.
\end{abstract}

\section{Commentary}

External or subcomissural annuloplasty in bicuspid aortic valve repair: which is better?

Running head: Bicuspid aortic valve repair

Metesh Acharya MBBS MD(Res) ${ }^{1}$, Hiwa Sherzad MD ${ }^{1}$, Sven ZCP Tan ${ }^{2}$, Mohamad Bashir MD²,

Giovanni Mariscalco MD PhD ${ }^{1}$

1. Department of Cardiac Surgery, Glenfield Hospital, Leicester, United Kingdom.

2. Barts and The London School of Medicine and Dentistry, Queen Mary University of London, United Kngdom

3. Vascular and Endovascular Surgery, Health Education and Improvement Wales, Nantgarw CF15 7QQ.

Sources of Funding: None

\section{Correspondence}

Giovanni Mariscalco MD PhD

Department of Cardiac Surgery, Glenfield Hospital, Groby Road, Leicester, LE3 9QP, United Kingdom

E-mail: giovanni.mariscalco@uhl-tr.nhs.uk

Tel: +44 (0) 3003031573 


\begin{abstract}
Patients with a bicuspid aortic valve (BAV) are at increased risk of valvular regurgitation compared to their counterparts with a tri-leaflet aortic valve. There is now increasing emphasis to offer BAV repair to mitigate the risks of prosthesis-related complications, including thromboembolism, haemorrhage and endocarditis, as well as structural valve deterioration and future re-operation with conventional valve replacement, particularly in younger populations. Furthermore, over the preceding two decades, our greater understanding of the functional anatomy of the BAV, pathophysiological mechanisms of BAV insufficiency and the development of a functional classification of aortic regurgitation have significantly contributed to the evolution of aortic valve reconstructive surgery. In this commentary, we discuss a recent article from the Journal of Cardiac Surgery comparing external annuloplasty and subcommissural annuloplasty as techniques for BAV repair.
\end{abstract}

128 words

\title{
Introduction
}

In this issue of the Journal, Jasinski and colleagues prospectively evaluate the single-centre outcomes of aortic annular stabilisation using either external annuloplasty (EA) or subcommisural annuloplasty (SCA) in bicuspid aortic valve (BAV) repair for aortic regurgitation (AR) alongside ascending aortic replacement ${ }^{1}$. 25 patients with type I BAV configuration that were assigned to each group underwent post-operative echocardiographic follow-up at 1 and 3 years, and morbidity and mortality outcomes were determined at 8 years. EA and SCA had comparable influences on echocardiographic indices of post-operative left ventricular reverse remodelling, and both techniques achieved similar significant reductions in aortic regurgitant jet width. However, a significant reduction in the aortic annulus diameter was only observed with the EA approach (27.5 $\pm 3 \mathrm{~mm}$ pre-operatively vs $24.1 \pm 2.6 \mathrm{~mm}$ post-operatively, $\mathrm{p}=0.002)$, and not with SCA $(27.7 \pm 4.7 \mathrm{~mm}$ pre-operatively vs $25.8 \pm 3.8 \mathrm{~mm}$ post-operatively, $\mathrm{p}=0.7)$. Furthermore, EA resulted in lower mean trans-valvular gradients compared to SCA $(7 \pm 4.0 \mathrm{mmHg}$ vs $12.6 \pm 4.6 \mathrm{mmHg}, \mathrm{p}=0.018)$. Whilst valvular re-operation was not indicated in any patient during the 8-year follow-up period, progression to grade [?]2 AR or trans-valvular gradient $>20 \mathrm{mmHg}$ was identified in 1/25 (4\%) patients and 6/25 (24\%) patients in the EA and SCA groups, respectively. The authors propose that EA is superior to SCA in terms of post-operative annular reduction, trans-valvular flow gradient and freedom from AR.

BAV represents the commonest congenital cardiac abnormality affecting 1-2\% of the general population ${ }^{2}$ and its association with valvular dysfunction, infective endocarditis, and aortopathy with proximal aortic aneurysm formation and a propensity for acute aortic events renders it a considerable health burden ${ }^{3,4}$. Furthermore, the incidence of AR in BAVs is greater than that in tri-leaflet aortic valves. Although valve replacement is traditionally considered the gold standard surgical management for severe AR, prosthesis-related complications including thromboembolism, haemorrhage and endocarditis, coupled with the cumulative risks of structural valve deterioration and attendant re-operative surgery remain a major concern, especially for younger patients. Survival after aortic valve replacement has been reported at $50 \%$ at 10 years ${ }^{5}$.

Strategies for isolated aortic valve repair followed the introduction of valve-sparing aortic root replacement procedures, first proposed as a remodelling technique in 1983 by Yacoub ${ }^{6}$ and subsequently with aortic valve reimplantation in 1992 by David ${ }^{7}$ which were conceived to treat aortic root dilatation without valve intervention. The aortic root complex, comprising the aortic annulus, aortic valve leaflets, the sinuses of Valsalva, and the sino-tubular junction, functions as a single integrated unit, and AR may originate from the disruption of any of its components ${ }^{8}$. Other pathologies causing AR therefore include leaflet prolapse, leaflet perforation and annular dilatation.

Over the preceding two decades, a more profound understanding of the functional anatomy of the BAV and the pathophysiological mechanisms underlying AR, the implementation of standardised nomenclature to describe the valvular defects encountered, ongoing refinements in operative techniques and the development of a functional classification of AR permitting a systematic approach to BAV repair ${ }^{9,10}$, have contributed greatly to the advancement of aortic valve reconstructive surgery. Recently, BAV repair received a class $2 \mathrm{~b}$ recommendation in the $2020 \mathrm{ACC} / \mathrm{AHA}$ valvular heart disease management guidelines, when performed by 
an experienced surgeon ${ }^{11}$.

Stabilisation of the aortic annulus is now well-established as a fundamental principle during repair of regurgitant $\mathrm{BAVs}^{12,13}$ for management of AR secondary to annular dilatation in the absence of structural valve disease. Uncorrected annular dilatation exceeding $25-29 \mathrm{~mm}$ is recognised as a predominant risk factor for repair failure ${ }^{14}$. A variety of surgical methods are available to stabilise the aortic annulus, including an external polytetrafluoroethylene (PTFE) suture $^{13}$ or flexible annuloplasty ring to correct ventriculo-aortic junction dilatation ${ }^{15}$, or internal placement of a Cabrol stitch ${ }^{16}$ (subcommissural annuloplasty, SCA), PTFE suture $^{17}$ or rigid annuloplasty ring ${ }^{18}$.

Due to the prevalence of functional aortic annular dilatation in regurgitant BAVs, annular stabilisation is critical to the durability of BAV repair. First described by Cabrol in $1966^{16}$, SCA is a relatively straightforward technique for aortic annular stabilisation whereby a horizontal mattress suture is placed in the subcommissural inter-leaflet triangle, reducing annular circumference and improving leaflet coaptation. Initially a popular approach, SCA produces asymmetric annular reduction only in the commissural zones of the aortic annulus, predisposing the remaining annulus to future dilatation requiring reintervention. Another potential drawback is that SCA may generate an unequal distribution of stress on the repaired BAV and risk recurrent $\mathrm{AR}^{19}$. This is further corroborated by the inferiority of SCA in patients with a larger aortic annulus compared to valve-sparing aortic root replacement, where the entire annulus is reinforced with pledgetted sutures. Indeed, some studies have highlighted SCA as a predictor of valve-related reoperation ${ }^{20-22}$ in both tri-leaflet aortic valves and BAVs, rendering the technique somewhat redundant. In contrast, EA utilises a continuous mattress suture placed through the aortic wall circumferentially around the entire aortic valve, passing through the nadirs of the coronary sinuses and imbricating the aortic wall as the suture is tensioned, thereby reducing the aortic annulus and promoting leaflet coaptation. Care must be taken to avoid injury to the coronary ostia. Rather than applying EA sutures, many groups now routinely perform valve-sparing reimplantation ${ }^{23}$, where the Dacron prosthesis provides complete annular stabilisation, although this is more technically challenging than SCA.

In the only available study directly comparing EA and SCA, Ko and colleagues analysed 20 patients with severe regurgitation in type I BAVs undergoing external subannular aortic ring (ESAR) constructed from a Dacron graft and 38 undergoing $\mathrm{SCA}^{19}$. They found that ESAR afforded greater reductions in annular dimensions and trans-valvular gradients than SCA, whilst both techniques resulted in $100 \%$ freedom from AR > grade 1 at a mean $9.2+-0.7$ months and $51.1+-38.5$ months in the SCA and ESAR groups, respectively.

In the present study by Jasinksi and colleagues ${ }^{1}$, SCA was performed using two braided $2 / 0$ sutures with pledgets to narrow two subcommissural triangles, whereas EA incorporated a line of interrupted braided $2 / 0$ sutures placed along the external perimeter of the aortic annulus, buttressed by a Dacron strip. Several additional valve preservation strategies were concomitantly employed, including prolapse repair, raphe excision and pericardial patch reconstruction of the aortic leaflets, although specific details are not provided, and their influence on the observed valve repair outcomes cannot be elucidated without additional analysis.

Nevertheless, Jasinski and colleagues are to be congratulated on the excellent outcomes achieved in their series of patients undergoing BAV repair with ascending aortic replacement. They have demonstrated good medium-term durability with freedom from AR progression beyond grade 2 with both EA and SCA techniques at $35.1+-3.6$ months, although this is significantly better at $96 \%$ with EA, compared to SCA at $76 \%$. EA also provides lower trans-valvular flow velocities and gradients than SCA. BAV repair with EA can be a safe and effective alternative to conventional valve replacement, conditional on judicious patient selection and the availability of surgical expertise for the geometric and functional normalisation of all components of the aortic valve and root. However, longer-term robustness of the repair with EA necessitates extended follow-up.

\section{References}

1. Jasinski M, Plonek T, Gocol R, Pysz P, Hudziak D, Wenzel-Jasińska I, et al. Bicuspid aortic valve repair with external or subcommissural annuloplasty - echocardiographic prospective trial. J Card 
Surg [ahead of press].

2. Hoffman JIE, Kaplan S. The incidence of congenital heart disease. J Am Coll Cardiol 2002;39:1890-900.

3. Fedak PW, Verma S, David TE, Leask RL, Weisel RD, Butany J. Clinical and pathophysiological implications of a bicuspid aortic valve. Circulation 2002;106:900-4.

4. Michelena HI, Khanna AD, Mahoney D, Margaryan E, Topilsky Y, Suri RM, et al. Incidence of aortic complications in patients with bicuspid aortic valves. JAMA 2011;306:1104-12.

5. Gaca JG, Clare RM, Rankin JS, Daneshmand MA, Milano CA, Hughes GC, et al. Risk-adjusted survival after tissue versus mechanical aortic valve replacement: a 23-year assessment. J Heart Valve Dis 2013;22:810-6.

6. Sarsam MA, Yacoub M. Remodeling of the aortic valve annulus. J Thorac Cardiovasc Surg 1993;105:435-8.

7. David TE, Feindel CM. An aortic valve-sparing operation for patients with aortic incompetence and aneurysm of the ascending aorta. J Thorac Cardiovasc Surg 1992;103:617-621.

8. El Khoury G, Vanoverschelde JL, Glineur G, Pierard F, Verhelst RR, Rubay J, et al. Repair of bicuspid aortic valves in patients with aortic regurgitation. Circulation 2006;114(1 Suppl):I610-6.

9. Boodhwani M, de Kerchove L, Glineur D, Poncelet A, Rubay J, Astarci P, et al. Repair-oriented classification of aortic insufficiency: impact on surgical techniques and clinical outcomes. J Thorac Cardiovasc Surg 2009;137:286-94.

10. Zakkar M, Youssefi P, Acar C, Khelil N, Debauchez M, Lansac E. Bicuspid aortic valve repair adapted to aortic phenotype. Ann Cardiothorac Surg 2019;8:401-10.

11. Otto CM, Nishimura RA, Bonow RO, Carebello BA, Erwin JP 3 $3^{\text {rd }}$, Gentile F, et al. 2020 ACC/AHA Guideline for the Management of Patients With Valvular Heart Disease: Executive Summary: A Report of the American College of Cardiology/American Heart Association Joint Committee on Clinical Practice Guidelines. Circulation 2021;143:e35-71.

12. Schäfers HJ, Raddatz A, Schmied W, Takahashi H, Miura Y, Kunihara T, et al. Reexamining remodeling. J Thorac Cardiovasc Surg 2015;149(2 Suppl):S30-6.

13. Schneider U, Aicher D, Miura Y, Schäfers HJ. Suture Annuloplasty in Aortic Valve Repair. Ann Thorac Surg 2016;101:783-5.

14. Schneider U, Hofmann C, Aicher D, Takahashi H, Miura Y, Schäfers HJ. Suture Annuloplasty Significantly Improves the Durability of Bicuspid Aortic Valve Repair. Ann Thorac Surg 2017;103:505-510.

15. Lansac E, Di Centa I, Sleilaty G, Lejeune S, Berrebi A, Zacek P, et al. Remodeling root repair with an external aortic ring annuloplasty. J Thorac Cardiovasc Surg 2017;153:1033-42.

16. Cabrol A, Cabrol C, Guiradon G, Bertrand M. Treatment of aortic insufficiency by means of aortic annuloplasty. Arch Mal Coeur Vaiss 1966;59:1305-12.

17. Schöllhorn J, Rylski B, Beyersdorf F. Aortic valve annuloplasty: new single suture technique. Ann Thorac Surg 2014;97:2211-3.

18. Gocoł R, Jasiński, Hudziak D, Bis J, Żak A, Duraj P, et al. Surgical correction of aortic regurgitation using a HAART 300 rigid aortic ring: A novel method to standardize aortic valve repair. Cardiol J 2019;26:799-801.

19. Ko H, Bavaria JE, Habertheuer A, Augoustides JG, Siki MA, Freas M, et al. Functional Outcomes of Type I Bicuspid Aortic Valve Repair With Annular Stabilization: Subcommissural Annuloplasty Versus External Subannular Aortic Ring. Ann Thorac Surg 2019;107:68-75.

20. Jasinski MJ, Kosiorowska K, Gocol R, Jasinski J, Nowicki R, Bielicki G, et al. Bicuspid aortic valve repair: outcomes after 17 years of experience. Eur J Cardiothorac Surg 2021;60:1053-1061.

21. Navarra E, Khoury GE, Glineur D, Boodhwani M, Van Dyck M, Vanoverschelde JL, et al. Effect of annulus dimension and annuloplasty on bicuspid aortic valve repair. Eur J Cardiothorac Surg 2013;44:316-23.

22. Aicher D, Langer F, Adam O, Tscholl D, Lausberg H, Schäfers HJ. Cusp repair in aortic valve reconstruction: does the technique affect stability? J Thorac Cardiovasc Surg 2007;134:1533-9.

23. El Khoury G, de Kerchove L. Principles of aortic valve repair. J Thorac Cardiovasc Surg 2013;145(3 Suppl):S26-9. 